\title{
Qualifizierung von Beschäftigten in einfachen Tätigkeiten und Fachkräftebedarf
}

\author{
Lutz Bellmann • Sandra Dummert • Margit Ebbinghaus • Elisabeth M. Krekel • \\ Ute Leber
}

Online publiziert: 1. Juli 2015

(C) Die Autor(en) 2015. Dieser Artikel ist auf Springerlink.com mit Open Access verfügbar.

Zusammenfassung Bei der Suche nach Lösungen für den sich bereits heute abzeichnenden Fachkräftemangel wurden Beschäftigte mit einfachen Tätigkeiten bisher vernachlässigt. Mit einem Anteil von rd. 20\% an allen Beschäftigten stellt diese Personengruppe allerdings ein nicht unerhebliches Qualifizierungspotenzial dar. Gegenstand des nachfolgenden Beitrags ist die Frage, ob erwartete bzw. aktuell bestehende Schwierigkeiten bei der Besetzung von Fachkräftestellen dazu führen, dass Betriebe verstärkt Beschäftigte in einfachen Tätigkeiten in Weiterbildung einbeziehen. Die Analysen zeigen, dass, neben anderen Merkmalen, insbesondere auch antizipierte Fachkräfteengpässe Treiber der betrieblichen Weiterbildungsbeteiligung von Beschäftigten in einfachen Tätigkeiten sind.

Schlüsselwörter Weiterbildung · Fachkräftemangel $\cdot$ Beschäftigte in einfachen Tätigkeiten

Prof. Dr. E. M. Krekel $(\bowtie) \cdot$ M. Ebbinghaus

BIBB - Bundesinstitut für Berufsbildung,

Robert-Schuman-Platz 3,

53175 Bonn, Deutschland

E-Mail: krekel@bibb.de

M. Ebbinghaus

E-Mail: Ebbinghaus@bibb.de

Prof. Dr. L. Bellmann · S. Dummert · Dr. U. Leber

Institut für Arbeitsmarkt- und Berufsforschung (IAB) der Bundesagentur für Arbeit (BA),

Regensburger Straße 104,

90478 Nürnberg, Deutschland

E-Mail: Lutz.Bellmann@iab.de

S. Dummert

E-Mail: Sandra.Dummert@iab.de

Dr. U. Leber

E-Mail: Ute.Leber@iab.de 
Abstract In the search for strategies to combat skills shortages, employees with simple tasks have often not been focused on. However, making up about $20 \%$ of all employees, this group of workers represents an important qualification potential. It is the purpose of our paper to investigate the question whether expected or existing difficulties in filling vacancies for skilled personnel increase the training activities of firms towards employees in low-skilled jobs. Our analyses show that expected skills shortages are, besides other factors, one driver of the training participation of employees in low-skilled jobs.

Keywords workers with simple tasks · participation in further education · demand for skilled workers

\section{Ausgangslage}

Gerade in konjunkturell guten Zeiten, in denen der betriebliche Personalbedarf besonders hoch ist, wird das Thema „Fachkräftemangel“ intensiv diskutiert. Vorliegende Untersuchungen zeigen, dass bereits heute zahlreiche Betriebe vor Rekrutierungsschwierigkeiten stehen. Betroffen sind vorrangig klein- und mittelständische Betriebe sowie Betriebe mit einem Fachkräftebedarf in technischen und elektrotechnischen Berufen sowie in Berufen des Gesundheitswesens (vgl. u. a. Bundesministerium für Wirtschaft und Technologie 2013; Dummert et al. 2014, S. 61 ff.; Czepek et al. 2015, S. 64 ff.). Engpässe im Fachkräfteangebot sind dabei jedoch kein vorübergehendes Phänomen. Vielmehr ist abzusehen, dass sich der Mangel an Fachkräften auch losgelöst von der konjunkturellen Entwicklung ausweiten wird. Dafür spricht zunächst die demografische Entwicklung, durch die deutlich mehr Personen aus dem Erwerbsleben ausscheiden als neu hinzukommen. Aufgrund der Bildungsexpansion verteilt sich der negative Saldo allerdings nicht gleichmäßig über die verschiedenen Qualifikationsstufen. Aktuellen Projektionen zufolge werden mittelfristig sogar „,immer mehr akademisch ausgebildete Personen in das Erwerbsleben eintreten als ausscheiden“ (Maier et al. 2014, S. 5), sodass der Rückgang der Erwerbspersonen ausschließlich zulasten der einfachen und mittleren Qualifikationsebenen gehen wird (ebd.). Da dieselben Projektionen zudem darauf verweisen, dass auch der Bedarf an Arbeitskräften ohne Berufsabschluss stark rückläufig sein wird, nicht jedoch der an Arbeitskräften mit einer abgeschlossenen Berufsausbildung (ebd.), dürfte die Deckung des Fachkräftebedarfs auf der mittleren Qualifikationsebene eine der zentralen Herausforderungen des Arbeitsmarktes werden.

Um dieser Herausforderung zu begegnen, kommt neben einer erhöhten Zuwanderung und einer weiter steigenden Erwerbsquote insbesondere der Erschließung vorhandener Qualifizierungsreserven eine große Bedeutung zu. Die bislang in diese Richtung angestoßenen Maßnahmen richten sich besonders auf die Nachqualifizierung von Personen ohne formalen Berufsabschluss. So will die Bundesregierung ,gezielt in die Nachqualifizierung junger Erwachsener ohne Berufsabschluss investieren" (Koalitionsvertrag zwischen CDU, CSU und SPD, 18. Legislaturperiode, S. 66), damit Erwachsene auch zu späteren Zeitpunkten noch einen Berufsabschluss erwerben können. In diesem Zusammenhang hat auch die Bundesagentur für Arbeit 
(BA) im Februar 2013 ihre Initiative „Ausbildung wird was - Spätstarter gesucht“ gestartet. Bis 2016 sollen 100.000 junge Menschen zwischen 25 und 35 Jahren dazu motiviert werden, einen erneuten Anlauf für eine Ausbildung zu nehmen. Ähnliche Ziele wurden auch mit der Förderinitiative ,Abschlussorientierte modulare Nachqualifizierung" verfolgt, die als Bestandteil des Strukturprogramms „Perspektive Berufsabschluss" explizit auf das Nachholen eines Berufsabschlusses ausgerichtet war (Bundesministerium für Bildung und Forschung 2013, S. 52 f.). Andere Initiativen fokussieren zudem auf den Erhalt bzw. Ausbau der Beschäftigungsfähigkeit gering qualifizierter und älterer Arbeitskräfte, wie beispielsweise das Programm „Weiterbildung Geringqualifizierter und beschäftigter Älterer in Unternehmen“" (WeGebAU) der BA.

Beschäftigte mit einfachen Tätigkeiten wurden in empirischen Studien indes bislang kaum als „Qualifikationspotenzial“ zur Begegnung von Fachkräfteengpässen in den Blick genommen. Im vorliegenden Beitrag wird dieser Aspekt aus betrieblicher Perspektive aufgegriffen. Es wird untersucht, inwieweit Betriebe Beschäftigte in einfachen Tätigkeiten an Weiterbildung beteiligen und welche Rolle hierfür, neben anderen Faktoren, Schwierigkeiten bei der Rekrutierung von Fachkräften spielen. Konkret wird die Frage untersucht, ob erwartete bzw. aktuell bestehende Schwierigkeiten bei der Besetzung von Fachkräftestellen dazu führen, dass Betriebe (auch) verstärkt Beschäftigte in einfachen Tätigkeiten in Weiterbildung einbeziehen. Vorab werden eine Einordnung einfacher Tätigkeiten und eine Einbettung der Fragestellung in den Weiterbildungsdiskurs vorgenommen.

\section{Einordnung einfacher Tätigkeiten}

Der Begriff „einfache Tätigkeiten“ (Synonym: Einfacharbeit) steht meist für Stellen bzw. Arbeiten, die keine formale Berufsausbildung erfordern. Das bedeutet aber nicht zwangsläufig, dass die Stelleninhaber nicht formal qualifiziert sind. Ebenso wenig sind „einfache Tätigkeiten“ mit Arbeiten gleichzusetzen, die mit keinen oder nur sehr geringen Qualifikationsanforderungen einhergehen (Weber und Kretschmer 2012). Zwar sind einfache Tätigkeiten nach wie vor vielfach durch einen hohen Anteil standardisierter, repetitiver Tätigkeiten gekennzeichnet (Clement 2007, S. 35), die nur einen geringen Komplexitätsgrad und wenig Handlungsautonomie aufweisen (Abel et al. 2009, S. 6 f.). Es lässt sich aber auch beobachten, dass die Technisierung der Arbeitswelt in Verbindung mit der Einführung prozessorientierter Formen der Arbeitsorganisation in vielen Unternehmen dazu geführt hat, dass der damit einhergehende ,,allgemeine Qualifikationsshift auch den Bereich der einfacheren Tätigkeiten erfasst hat" (Galiläer 2006, S. 4). Denn indem einfache Arbeitsplätze in komplexe Arbeitsprozesse eingebunden werden, stellen sie Anforderungen an Qualifikationen, die an der Schnittstelle zwischen einfacher und qualifizierter Tätigkeit liegen (Abel et al. 2014; Galiläer 2006, S. 5 f.; Weber und Kretschmer 2012, S. 40). Dies geht allerdings weniger mit einem „Upgrade“ zur Fachkräftetätigkeit einher als vielmehr mit der Herausbildung verschiedener Formen von Einfacharbeit, die sich u. a. in der Aufgabenvielfalt und den auf ihre Bearbeitung bezogenen Dispositionsspielräumen (z. B. Reihenfolge, Interaktionen) unterscheiden (Abel et al. 2009, S. 34 ff.). 


\section{Theoretische und empirische Bezüge}

Die Auseinandersetzung mit Fragen der beruflichen Weiterbildung von Beschäftigten mit einfachen Tätigkeiten kann sich entweder auf individuelle oder auf betriebliche Weiterbildungsaktivitäten beziehen. Im vorliegenden Beitrag konzentrieren wir uns auf die Beteiligung von Personen in einfachen Tätigkeiten an betrieblicher Weiterbildung.

Die bisherigen Befunde zur Beteiligung von Beschäftigen in einfachen Tätigkeiten an betrieblichen Weiterbildungsaktivitäten basieren überwiegend auf Studien, bei denen der Tätigkeitsstatus (einfache, qualifizierte, hoch qualifizierte Tätigkeit) als eine von mehreren Bestimmungsgrößen des betrieblichen Qualifizierungsverhaltens untersucht wurde. Diese verweisen konsistent darauf, dass „es in der betrieblichen Weiterbildung zu einer stark qualifikations- und arbeitsplatzabhängigen Weiterbildungsbeteiligung kommt“" (Käpplinger 2011, S. 55). Das äußert sich u. a. darin, dass sich das betriebliche Weiterbildungsengagement sehr viel stärker auf Beschäftigte mit qualifizierten als auf solche mit einfachen Tätigkeiten richtet (u. a. Stegmaier 2009; Leber 2009; Grunau 2012; Hartung 2013). Zudem wurde gezeigt, dass Beschäftigte mit einfachen Tätigkeiten dann über eine besonders geringe Weiterbildungsbeteiligung verfügen, wenn sie zusätzlich Merkmale aufweisen, die insgesamt mit einer geringen Weiterbildungsteilnahme einhergehen. Hierbei handelt es sich beispielsweise um eine atypische Beschäftigung wie Zeitarbeit, einen Migrationshintergrund oder das Alter (Bellmann et al. 2013).

Die Erklärung der (fremd-)selektiven Zugänge zu betrieblicher Weiterbildung wird in der Regel in den betrieblichen Nutzenkalkülen gesucht, für die primär auf den humankapitaltheoretischen Ansatz von Becker (1964) Bezug genommen wird (vgl. u. a. Düll und Bellmann 1999). Hiernach stellen betriebliche Qualifizierungsmaßnahmen eine Investition in die Produktivkraft der Beschäftigten dar, die sich längerfristig in höheren Erträgen niederschlagen soll. Da dies den Verbleib der Beschäftigten im Betrieb voraussetzt, sollten sich die betrieblichen Weiterbildungsaktivitäten auf spezifisch im Betrieb verwertbare Qualifikationen richten. Demgegenüber zeichnet sich das für einfache Tätigkeiten erforderliche Qualifikationsprofil - auch unter der Bedingung des oben ausgeführten ,allgemeinen Qualifikationsshifts“ - durch „unspezifische Basisqualifikationen“ (Köhler und Loudovici 2007, S. 27, zit. n. Abel et al. 2009, S. 11) aus, die leicht transferierbar sind, so dass Betriebe nur wenig Interesse daran haben sollten, in diese durch Weiterbildung zu investieren. Für andere Merkmale bzw. Beschäftigtengruppen, wie prekär oder ältere Beschäftigte, lässt sich aus der humankapitaltheoretischen Nutzenargumentation ableiten, dass hier die begrenzte Zeitspanne, in der Nutzen realisiert werden kann, ihrer Weiterbildungsbeteiligung aus betrieblicher Sicht entgegensteht.

Als alleiniger Bezugspunkt für die betrieblichen Weiterbildungsaktivitäten greift der humankapitaltheoretische Erklärungsansatz allerdings zu kurz. Denn auch wenn Beschäftige in einfachen Tätigkeiten nicht in gleichem Maße wie andere Beschäftigte an betrieblicher Weiterbildung beteiligt werden, so partizipieren sie gleichwohl daran. Nach Käpplinger (2011) sind betriebliche Weiterbildungsentscheidungen ein Resultat „sozialer Aushandlungs- und Abstimmungsprozesse“ (S. 57), in die unterschiedliche „Rationalitäten und Motive“ (S. 56) einfließen. Das unterstreichen 
Befunde, nach denen Betriebsräte und die Tarifbindung von Betrieben einen positiven Einfluss auf die Weiterbildungsquote haben (Düll und Bellmann 1999). Eine weitere „Rationalität“ kann auch das Kostenkalkül bei der Deckung von Qualifikationsbedarfen sein. Nach dem transaktionskostentheoretischen Erklärungsansatz (Williamson 1990) ist für dieses Kalkül vor allem die Verfügbarkeit der nachgefragten Qualifikation am externen Arbeitsmarkt von Bedeutung. Da diese den Aufwand und zugleich die Erfolgsaussichten des Suchprozesses beeinflusst, sollte das Kostenkalkül umso mehr zugunsten einer internen Bedarfsdeckung ausfallen, je seltener die vom Betrieb benötigte Qualifikation auf dem externen Arbeitsmarkt zu finden ist. Entsprechend steht zu erwarten, dass Betriebe Beschäftigte mit einfachen Tätigkeiten mit zunehmenden (Fachkräfte-)Engpässen am Arbeitsmarkt vermehrt in ihre Weiterbildungsaktivitäten einbeziehen. In diese Richtung deuten die Befunde einiger aktuellerer Studien. So vertreten der 2011 durchgeführten Weiterbildungserhebung des Instituts der deutschen Wirtschaft (IW) zufolge gut vier von zehn Unternehmen die Ansicht, dass An- und Ungelernte dank Weiterbildung qualifizierte Tätigkeiten ausüben können (Seyda und Werner 2012). Doch trotz dieser grundsätzlich positiven Haltung der Betriebe weisen die Autoren darauf hin, dass die Weiterbildungsbeteiligung Geringqualifizierter nach wie vor hinter der Höherqualifizierter zurückbleibe. Die Studie von Troltsch (2013) macht deutlich, dass die Bereitschaft der Betriebe zur Nachqualifizierung junger An- und Unqualifizierter wie auch zur Weiterqualifizierung Älterer jeweils dann größer ist, wenn Schwierigkeiten bei der Fachkräfterekrutierung nicht nur erwartet, sondern bereits erfahren werden. Ebenso zeigt auch die Untersuchung von Elsholz (2007), dass die interne Sicherung des Fachkräftepotenzials und die Arbeitskräftebindung aus Unternehmenssicht wesentliche Motive zur Weiterbildung Geringqualifizierter sind.

Den zuletzt referierten Studien ist gemeinsam, dass sie sich der Bedeutung von Fachkräfteengpässen für die Erschließung vorhandenen Qualifikationspotenzials über das Qualifikationsniveau der Beschäftigten angenähert haben. In der vorliegenden Arbeit wird hingegen eine Annäherung über das Tätigkeitsniveau vorgenommen.

\section{Gegenstand und Datenbasis der empirischen Analysen}

Nach den Daten des IAB-Betriebspanels machten einfach Beschäftigte im Jahr 2013 rund $21 \%$ aller in Deutschland Beschäftigten aus und waren in etwa $48 \%$ der Betriebe zu finden. Sie stellen damit ein nicht unerhebliches Qualifizierungspotenzial dar, wobei angenommen werden kann, dass Betriebe dieses Potenzial vor allem dann erschließen, wenn sie Fachkräfte brauchen, die sie über den externen Arbeitsmarkt nicht gewinnen können.

Diesem Aspekt wollen wir in den nachfolgenden Analysen nachgehen. Konkret soll die Frage untersucht werden, ob erwartete bzw. aktuell bestehende Schwierigkeiten bei der Besetzung von Fachkräftestellen dazu führen, dass Betriebe (auch) verstärkt Beschäftigte in einfachen Tätigkeiten in Weiterbildung einbeziehen. Für die Untersuchung der Fragestellung werden Daten aus der Erhebungswelle 2013 des IAB-Betriebspanels herangezogen (siehe Datenbasis). Diese werden zunächst deskriptiv und daran anschließend multivariat ausgewertet. 
Datenbasis Beim IAB-Betriebspanel handelt es sich um eine jährlich durchgeführte Befragung von rund 16.000 Betrieben aller Branchen und Größenklassen, die mindestens einen sozialversicherungspflichtig Beschäftigten haben. Die Befragungen sind jeweils als Mehr-Themen-Befragungen angelegt und werden durch TNS Infratest Sozialforschung im Auftrag des IAB durchgeführt (für weitere Informationen zum IAB-Betriebspanel vgl. Ellguth et al. 2014). Folgende Kenngrößen spielen in unserer Analyse eine Rolle:

Zur Beschreibung der Weiterbildung ziehen wir die Weiterbildungsbeteiligung und die Weiterbildungsquote heran. Der Weiterbildungsbegriff wird im IAB-Betriebspanel relativ weit gefasst und bezieht sich neben formalen Maßnahmen, wie kursförmigen Schulungen, auch auf weichere Formen, wie das selbstgesteuerte Lernen am Arbeitsplatz und die Beteiligung an Qualitätszirkeln. Die Weiterbildungsbeteiligung bezieht sich darauf, ob ein Betrieb in der ersten Jahreshälfte weiterbildungsaktiv war. Als weiterbildungsaktiv gelten Betriebe, die Beschäftigte für die Teilnahme an Weiterbildung freigestellt oder die Kosten der Weiterbildung ganz oder teilweise übernommen haben. Die Weiterbildungsquote bezieht sich auf den Anteil der Beschäftigten, die in der ersten Jahreshälfte an Weiterbildung teilgenommen haben, an allen Beschäftigten eines Betriebes. Teilweise wird diese Größe auch als Teilnahmequote bezeichnet.

Der Fachkräftebedarf wird zum einen anhand der Frage, ob die Betriebe für die nahe Zukunft Schwierigkeiten bei der Fachkräfterekrutierung erwarten, operationalisiert. Zum anderen betrachten wir aber auch einen bereits bestehenden Fachkräftebedarf in Form des Vorliegens unbesetzter Stellen für Fachkräfte.

Beschäftigte für einfache Tätigkeiten schließlich kennzeichnen Mitarbeiter für Tätigkeiten, die keine Berufsausbildung erfordern. Fachkräfte hingegen stehen für Mitarbeitende für qualifizierte Tätigkeiten, die eine abgeschlossene Berufsausbildung oder ein (Fach-)Hochschulstudium erfordern.

\section{Deskriptive Ergebnisse: Weiterbildungsbeteiligung von Beschäftigten in einfachen Tätigkeiten}

Die Ergebnisse des IAB-Betriebspanels zeigen, dass sich im ersten Halbjahr 2013 zwölf Prozent der Betriebe mit mindestens einem Beschäftigten in einfachen Tätigkeiten an der Weiterbildung dieser Personengruppe beteiligt haben. Die Teilnahmequote der Beschäftigten in einfachen Tätigkeiten an Weiterbildung lag bei $16 \%$, d. h. von allen einfach Beschäftigten partizipierte knapp jeder Fünfte an Weiterbildungsmaßnahmen. In Ostdeutschland erreichte die Quote einen Wert von 21\% und lag damit um sechs Prozentpunkte über der in Westdeutschland. Die Differenzierung nach Betriebsgröße legt offen, dass sich die Weiterbildungsquote für die Beschäftigten in einfachen Tätigkeiten mit zunehmender Beschäftigtenzahl erhöht. Beschäftigte, die qualifizierte Tätigkeiten ausüben, ${ }^{1}$ nahmen wesentlich häufiger an Weiterbildungen teil als Beschäftigte in einfachen Tätigkeiten. So lag die Weiterbildungsquote der

\footnotetext{
${ }^{1}$ Als qualifizierte Tätigkeiten sind hier alle Tätigkeiten zusammengefasst, die in der Regel eine abgeschlossene duale Berufsausbildung, eine vergleichbare Berufsausbildung, einen Meister-, Techniker- oder vergleichbaren Abschluss oder ein (Fach-)Hochschulstudium erfordern.
} 


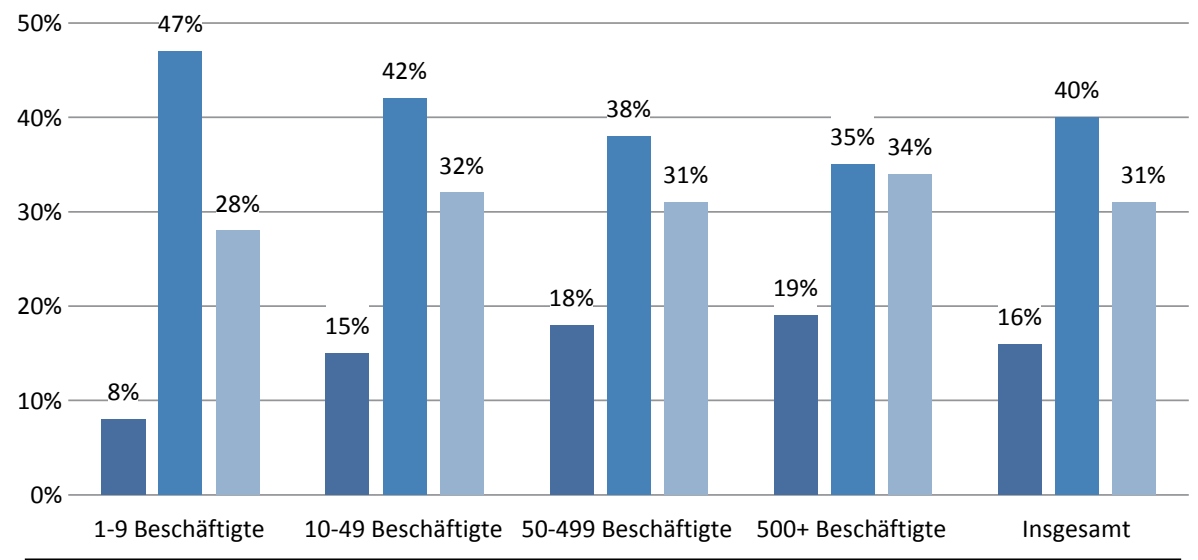

— Beschäftigte in einfachen Tätigkeiten $\quad$ Beschäftigte in qualifizierten Tätigkeiten $\quad$ Alle Beschäftigten

Abb. 1 Weiterbildungsquote von Beschäftigten in einfachen und qualifizierten Tätigkeiten nach Betriebsgröße 2013. (Angaben in \%) (Quelle: IAB-Betriebspanel 2013)

Beschäftigten in qualifizierten Tätigkeiten im Jahr 2013 in Deutschland 24 Prozentpunkte höher als die der einfach Beschäftigten (vgl. Abb. 1).

Die Weiterbildung einfach Beschäftigter spielte insbesondere in den Bereichen der Produktionsgüterindustrie, der Nahrungs- und Genussmittelherstellung sowie dem Gesundheits- und Sozialwesen eine überdurchschnittliche Rolle. Während durchschnittlich zwölf Prozent der Betriebe mit mindestens einem einfach Beschäftigten diese in Weiterbildungsmaßnahmen einbezogen, lag der Anteil in den genannten Wirtschaftszweigen acht bis zwölf Prozentpunkte höher. Betrachtet man zusätzlich die Intensität der Weiterbildung anhand der Weiterbildungsquoten, wird erneut deutlich, dass die Weiterbildung einfach Beschäftigter insbesondere im Wirtschaftszweig Gesundheits- und Sozialwesen einen hohen Stellenwert innehat. $34 \%$ der Beschäftigten in einfachen Tätigkeiten im Gesundheitssektor nahmen im ersten Halbjahr 2013 an Weiterbildung teil, gefolgt von der Produktionsgüterindustrie (23\%) und dem Einzelhandel (19\%).

Fachkräfteengpässe spielen in den Betrieben eine stetig zunehmende Rolle. So erwartete im Jahr 2012 ein Drittel der Betriebe mit mindestens einem einfach Beschäftigten in der Belegschaft für die folgenden zwei Jahre Schwierigkeiten bei der Besetzung von Fachkräftestellen. 2006 lag der Anteil noch bei 17\%. In den Wirtschaftszweigen Information und Kommunikation (52\%), der Investitionsund Gebrauchsgüterindustrie (42\%) und dem Baugewerbe (40\%) erwarteten die Betriebe mit einfachen Beschäftigten am häufigsten Probleme bei der Gewinnung geeigneter qualifizierter Beschäftigter. Differenziert man zusätzlich nach Betriebsgrößenklassen, zeigt sich, dass Großbetriebe im Jahr 2012 häufiger Rekrutierungsprobleme erwarteten als kleinere und mittlere Betriebe.

Betrachtet man nicht die für die nahe Zukunft erwarteten, sondern die gegenwärtigen Probleme bei der Fachkräfterekrutierung (hier operationalisiert durch unbesetzte Stellen für Fachkräfte im ersten Halbjahr 2013), so berichteten nur sechs Prozent der deutschen Betriebe mit mindestens einem einfach Beschäftigten von derartigen 
Abb. 2 Betriebliche Weiterbildungsbeteiligung und Weiterbildungsquote der einfach Beschäftigten bei erwarteten Fachkräfteproblemen (Angaben in \%). (Quelle: IAB-Betriebspanel 2012 und 2013)

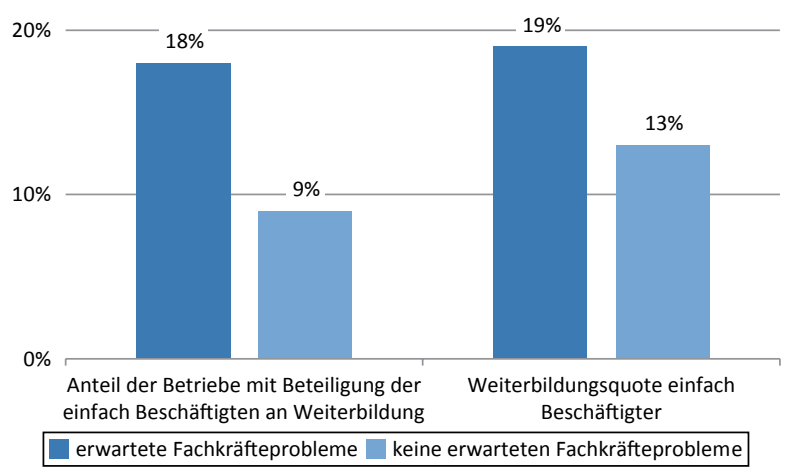

Schwierigkeiten (2007: 4\%). Auch hier ist ein Anstieg mit der Betriebsgröße zu erkennen. Unbesetzte Stellen für Fachkräfte waren im Jahr 2013 in den Wirtschaftszweigen der Unternehmensnahen Dienstleistungen, der Investitions- und Gebrauchsgüterindustrie und des Gesundheits- und Sozialwesens mit jeweils acht Prozent der Betriebe am weitesten verbreitet.

Haben Betriebe Fachkräfteengpässe, so geht dies mit Unterschieden in der Weiterbildung einfach Beschäftigter einher. So bildeten 2013 18\% der Betriebe mit mindestens einem einfach Beschäftigten, die im Jahr 2012 (also im Vorjahr) für die folgenden zwei Jahre Schwierigkeiten bei der Stellenbesetzung für Fachkräftestellen erwartet haben, ihre einfach Beschäftigten weiter. Ging der Betrieb hingegen nicht von Problemen hinsichtlich der Fachkräfterekrutierung aus, beteiligten sich lediglich 9\% der Betriebe an der Weiterbildung dieser Personengruppe. Die Weiterbildungsquote der Beschäftigten, die einfache Tätigkeiten ausüben, liegt in den Betrieben mit erwarteten Problemen bei 19\%. In Betrieben, die wiederum in 2012 keine Fachkräfteschwierigkeiten antizipiert haben, nahmen im Folgejahr $13 \%$ der einfach Beschäftigten an Qualifizierungsmaßnahmen teil (vgl. Abb. 2).

Ebenso wird deutlich, dass $24 \%$ der Betriebe mit aktuellen Problemen bei der Besetzung von Stellen für qualifizierte Tätigkeiten ihre einfach Beschäftigten 2013 in Weiterbildung einbezogen haben. Bei den Betrieben ohne unbesetzte Fachkräftestellen waren es hingegen lediglich 12\%. Die Weiterbildungsquote der einfach Beschäf-

Abb. 3 Betriebliche Weiterbildungsbeteiligung und Weiterbildungsquote der einfach Beschäftigten bei unbesetzten Stellen für Fachkräfte (Angaben in \%). (Quelle: IAB-Betriebspanel 2013)

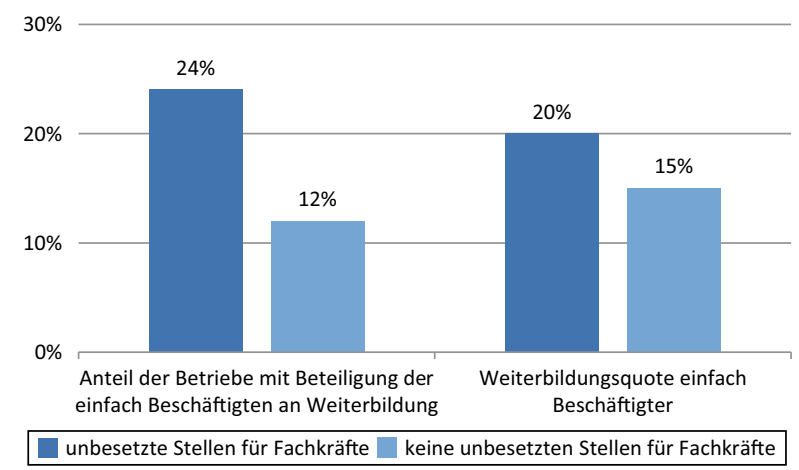


tigten lag in Betrieben mit offenen Stellen für Fachkräfte bei $20 \%$ und in Betrieben ohne solche vakanten Arbeitsplätze bei $15 \%$ (vgl. Abb. 3).

Die deskriptiven Befunde geben somit erste Hinweise darauf, dass Beschäftigte in einfachen Tätigkeiten häufiger an Weiterbildung teilnehmen, wenn ihr Betrieb Stellenbesetzungsprobleme erwartet oder bereits hat. Insgesamt gesehen nehmen Beschäftigte in einfachen Tätigkeiten jedoch sehr viel seltener an Aktivitäten der Weiterbildung teil als Beschäftigte in qualifizierten Tätigkeiten. Ob sich dies auch multivariat bestätigen lässt, wird im folgenden Abschnitt untersucht.

\section{Multivariate Ergebnisse: Haben Stellenbesetzungsschwierigkeiten für Fachkräfte einen Effekt auf die Weiterbildungsbeteiligung von Beschäftigten in einfachen Tätigkeiten?}

Um einen näheren Einblick in die Frage zu gewinnen, welche Betriebe stärker in der Weiterbildung ihrer einfachen Beschäftigten engagiert sind als andere und welche Rolle dabei einzelne Determinanten wie Stellenbesetzungsschwierigkeiten für Fachkräfte spielen, werden im Folgenden die Ergebnisse multivariater Analysen präsentiert. Diese erlauben es, den Einfluss eines Faktors auf das Weiterbildungsverhalten von Betrieben unter Kontrolle anderer Größen zu ermitteln, also abzuschätzen, inwieweit von den jeweiligen Betriebscharakteristika eigenständige Effekte auf das betriebliche Weiterbildungsverhalten ausgehen. Die Basis der multivariaten Analysen bilden dabei all jene Betriebe, die überhaupt Tätigkeiten für einfach Beschäftigte haben. Zunächst werden anhand von Probitschätzungen die Einflussfaktoren des Angebots an Weiterbildung für einfach Beschäftigte untersucht, ohne auf dessen Intensität einzugehen (Modelle 1 und 2). Die Intensität, d. h. der Umfang der Weiterbildungsbeteiligung, wird dann in weiteren Schätzungen berücksichtigt, indem die Weiterbildungsquote der einfach Beschäftigten als abhängige Variable herangezogen wird (Modelle 3 und 4). Da die abhängige Variable „Weiterbildungsquote einfach Beschäftigter" sowohl den Wert Null im Falle keiner betrieblichen Einbeziehung von einfach Beschäftigten in Weiterbildung ${ }^{2}$ als auch den Wert Eins bei einer vollständigen Teilnahme aller einfach Beschäftigten an Qualifizierungsmaßnahmen annehmen kann, werden links- und rechtszensierte Tobit-Modelle als Analyseverfahren geschätzt (vgl. Angrist und Pischke 2009, S. 99 ff.; Düll und Bellmann 1999, S. 77).

Als erklärende Variablen (Einflussfaktoren) verwenden wir verschiedene Faktoren, die sich im Wesentlichen aus den oben dargestellten theoretischen Erklärungsansätzen ergeben. Dabei handelt es sich um Merkmale der Personalstruktur, den Kontext der Arbeitsbeziehungen, technische Neuerungen, die Institutionalisierung der Personalarbeit, die Betriebsgröße sowie die Branchenzugehörigkeit (Tab. 1).

Die Branchenzugehörigkeit wird durch Dummies und die Betriebsgröße in logarithmierter Form in den Schätzungen berücksichtigt, um den nicht-linearen Zusammenhang abbilden zu können. Das besondere Augenmerk liegt allerdings auf den erwarteten und den tatsächlichen Schwierigkeiten bei der Besetzung von Fachkräfte-

\footnotetext{
${ }^{2}$ Das sind entsprechend die Betriebe, die im Probit-Modell die Referenzkategorie „,keine Weiterbildung für einfach Beschäftigte“ bilden.
} 
Tab. 1 Erklärende Variablen

\begin{tabular}{|c|c|c|}
\hline & Operationalisierung & $\begin{array}{l}\text { Erwarteter } \\
\text { Einfluss }\end{array}$ \\
\hline \multicolumn{3}{|l|}{ Stellenbesetzungsschwierigkeiten für Fachkräfte } \\
\hline Erwartetes Fachkräfteproblem im Vorjahr & Dummyvariable $(1=\mathrm{ja})$ & + \\
\hline Unbesetzte Stellen für qualifizierte Tätigkeiten & Dummyvariable $(1=$ ja $)$ & + \\
\hline \multicolumn{3}{|l|}{ Personalstruktur } \\
\hline Anteil von Frauen an allen Beschäftigten & Anteilsvariable ( 0 bis 1$)$ & $+/-^{\mathrm{a}}$ \\
\hline Anteil Beschäftigter für qualifizierte Tätigkeiten & Anteilsvariable ( 0 bis 1$)$ & $+/-^{\mathrm{a}}$ \\
\hline Anteil Beschäftigter für einfache Tätigkeiten & Anteilsvariable ( 0 bis 1$)$ & + \\
\hline \multicolumn{3}{|l|}{ Technische Ausstattung/Investitionen } \\
\hline Technische Ausstattung & $\begin{array}{l}\text { Dummyvariable }(1=\text { sehr } \\
\text { modern/modern })\end{array}$ & + \\
\hline Investitionen in Produktionsanlagen & Dummyvariable $(1=\mathrm{ja})$ & + \\
\hline $\begin{array}{l}\text { Investitionen in Informations-/Kommunikationstechnik } \\
\text { (IKT) }\end{array}$ & Dummyvariable $(1=$ ja $)$ & + \\
\hline \multicolumn{3}{|l|}{ Arbeitsbeziehungen } \\
\hline Existenz eines Betriebs- oder Personalrats & Dummyvariable $(1=\mathrm{ja})$ & + \\
\hline Tarifbindung & Dummyvariable $(1=\mathrm{ja})$ & + \\
\hline \multicolumn{3}{|l|}{ Formalisierungsgrad der Weiterbildung } \\
\hline schriftlich fixierte Pläne für die Personalentwicklung & Dummyvariable $(1=\mathrm{ja})$ & + \\
\hline Ausbildungsbetrieb & Dummyvariable $(1=2009 \mathrm{ja})$ & + \\
\hline Beschäftigtenzahl & Logarithmiert & + \\
\hline
\end{tabular}

${ }^{\mathrm{a} E s}$ existieren hier unserer Meinung nach sowohl Argumente für einen positiven als auch einen negativen Effekt

stellen als weitere potenzielle Einflussgrößen auf die Weiterbildung von Beschäftigten in einfachen Tätigkeiten. Modell 1 und 3 weisen jeweils die Werte für die erwarteten und Modell 2 und 4 für die tatsächlichen Stellenbesetzungsprobleme aus. ${ }^{3}$ Dahinter steht die Idee, dass einfach Beschäftigte ein wichtiges Potenzial zur Deckung von Fachkräfteengpässen darstellen können, indem sie so weiterqualifiziert werden, dass sie höherwertigere bzw. qualifiziertere Tätigkeiten ausüben können.

Die Ergebnisse der Schätzungen sind in Tab. 2 dargestellt. Dort sind neben den Koeffizienten auch die marginalen Effekte ausgewiesen, die Aussagen nicht nur zur Richtung, sondern auch zur Stärke des jeweiligen Effektes zulassen. Von den von uns betrachteten Merkmalen der Personalstruktur geht ein statistisch signifikanter und zugleich von der Größe bedeutsamer Effekt insbesondere vom Anteil der einfach Beschäftigten, aber auch dem Anteil der Frauen aus. Je mehr einfache Beschäftigte und je mehr Frauen in einem Betrieb beschäftigt sind, desto größer ist zum einen die Wahrscheinlichkeit, dass ein Betrieb Weiterbildung für einfach Beschäftigte anbietet, zum anderen aber auch die Weiterbildungsintensität dieser Beschäftigtengruppe. Es ist anzunehmen, dass mit zunehmendem Anteil einfacher Beschäftigter an den Belegschaften auch deren Bedeutung im Prozess der betrieblichen Leistungserstellung sowie ihre relative Verhandlungsmacht steigt, was wiederum mit verbesserten

\footnotetext{
${ }^{3}$ Aufgrund von Multikollinearitätsproblemen werden nicht beide Variablen in einer Schätzung berücksichtigt.
} 
Tab. 2 Probit- und Tobit-Modelle der Weiterbildung einfach Beschäftigter. (Quelle: IAB-Betriebspanel 2013)

\begin{tabular}{|c|c|c|c|c|c|c|c|c|}
\hline & \multicolumn{4}{|c|}{$\begin{array}{l}\text { Weiterbildung einfach Beschäftigter } \\
\text { ja/nein (Probit) }\end{array}$} & \multicolumn{4}{|c|}{$\begin{array}{l}\text { Weiterbildungsquote einfach Beschäftig- } \\
\text { ter (Tobit) }\end{array}$} \\
\hline & \multicolumn{2}{|l|}{ Modell 1} & \multicolumn{2}{|l|}{ Modell 2} & \multicolumn{2}{|l|}{ Modell 3} & \multicolumn{2}{|l|}{ Modell 4} \\
\hline & Koeff. & $\begin{array}{l}\text { Marg. } \\
\text { Effekt }\end{array}$ & Koeff. & $\begin{array}{l}\text { Marg. } \\
\text { Effekt }\end{array}$ & Koeff. & $\begin{array}{l}\text { Marg. } \\
\text { Effekt }\end{array}$ & Koeff. & $\begin{array}{l}\text { Marg. } \\
\text { Effekt }\end{array}$ \\
\hline $\begin{array}{l}\text { Erwartetes Fach- } \\
\text { kräfteproblem im } \\
\text { Vorjahr }(1=\mathrm{ja})\end{array}$ & $\begin{array}{l}0,242 * * * \\
(0,041)\end{array}$ & $\begin{array}{l}0,065^{* * *} \\
(0,011)\end{array}$ & & & $\begin{array}{l}0,248 * * * \\
(0,047)\end{array}$ & $\begin{array}{l}0,013 * * * \\
(0,002)\end{array}$ & & \\
\hline $\begin{array}{l}\text { Unbesetzte } \\
\text { Stellen für qualifi- } \\
\text { zierte Tätigkeiten } \\
(1=\text { ja })\end{array}$ & & & $\begin{array}{l}0,174 * * * \\
(0,050)\end{array}$ & $\begin{array}{l}0,047 * * * \\
(0,014)\end{array}$ & & & $\begin{array}{l}0,181^{* * *} \\
(0,054)\end{array}$ & $\begin{array}{l}0,010 * * * \\
(0,003)\end{array}$ \\
\hline $\begin{array}{l}\text { Anteil von } \\
\text { Frauen an allen } \\
\text { Beschäftigten }\end{array}$ & $\begin{array}{l}0,416^{* * *} \\
(0,085)\end{array}$ & $\begin{array}{l}0,112^{* * *} \\
(0,023)\end{array}$ & $\begin{array}{l}0,374 * * * \\
(0,076)\end{array}$ & $\begin{array}{l}0,102 * * * \\
(0,021)\end{array}$ & $\begin{array}{l}0,564 * * * \\
(0,100)\end{array}$ & $\begin{array}{l}0,030 * * * \\
(0,005)\end{array}$ & $\begin{array}{l}0,517 * * * \\
(0,088)\end{array}$ & $\begin{array}{l}0,028 * * * \\
(0,005)\end{array}$ \\
\hline $\begin{array}{l}\text { Anteil Beschäftig- } \\
\text { ter für qualifizier- } \\
\text { te Tätigkeiten }\end{array}$ & $\begin{array}{c}0,191 \\
(0,229)\end{array}$ & $\begin{array}{c}0,051 \\
(0,061)\end{array}$ & $\begin{array}{c}0,253 \\
(0,207)\end{array}$ & $\begin{array}{c}0,069 \\
(0,056)\end{array}$ & $\begin{array}{c}0,538^{*} \\
(0,286)\end{array}$ & $\begin{array}{c}0,028^{*} \\
(0,015)\end{array}$ & $\begin{array}{l}0,592 * * \\
(0,254)\end{array}$ & $\begin{array}{l}0,032 * * \\
(0,014)\end{array}$ \\
\hline $\begin{array}{l}\text { Anteil Beschäf- } \\
\text { tigter für einfache } \\
\text { Tätigkeiten }\end{array}$ & $\begin{array}{l}1,510 * * * \\
(0,229)\end{array}$ & $\begin{array}{l}0,406 * * * \\
(0,061)\end{array}$ & $\begin{array}{l}1,544 * * * \\
(0,206)\end{array}$ & $\begin{array}{l}0,422 * * * \\
(0,056)\end{array}$ & $\begin{array}{l}1,629 * * * \\
(0,281)\end{array}$ & $\begin{array}{l}0,085^{* * *} \\
(0,015)\end{array}$ & $\begin{array}{l}1,634 * * * \\
(0,248)\end{array}$ & $\begin{array}{l}0,089 * * * \\
(0,014)\end{array}$ \\
\hline $\begin{array}{l}\text { Technische Aus- } \\
\text { stattung }(1=\text { sehr } \\
\text { modern/modern })\end{array}$ & $\begin{array}{c}0,064 \\
(0,041)\end{array}$ & $\begin{array}{c}0,017 \\
(0,011)\end{array}$ & $\begin{array}{c}0,048 \\
(0,037)\end{array}$ & $\begin{array}{c}0,013 \\
(0,010)\end{array}$ & $\begin{array}{c}0,085^{*} \\
(0,046)\end{array}$ & $\begin{array}{c}0,004 * \\
(0,002)\end{array}$ & $\begin{array}{c}0,062 \\
(0,042)\end{array}$ & $\begin{array}{c}0,003 \\
(0,002)\end{array}$ \\
\hline $\begin{array}{l}\text { Investitionen in } \\
\text { Produktionsan- } \\
\text { lagen }(1=\mathrm{ja})\end{array}$ & $\begin{array}{c}0,081 * \\
(0,045)\end{array}$ & $\begin{array}{c}0,022 * \\
(0,012)\end{array}$ & $\begin{array}{c}0,060 \\
(0,042)\end{array}$ & $\begin{array}{c}0,016 \\
(0,011)\end{array}$ & $\begin{array}{l}0,104 * * \\
(0,052)\end{array}$ & $\begin{array}{l}0,005 * * \\
(0,003)\end{array}$ & $\begin{array}{c}0,077 * \\
(0,047)\end{array}$ & $\begin{array}{c}0,004 * \\
(0,003)\end{array}$ \\
\hline $\begin{array}{l}\text { Investitionen in } \\
\text { IKT }(1=\mathrm{ja})\end{array}$ & $\begin{array}{l}0,145 * * * \\
(0,046)\end{array}$ & $\begin{array}{l}0,039 * * * \\
(0,012)\end{array}$ & $\begin{array}{l}0,186 * * * \\
(0,042)\end{array}$ & $\begin{array}{l}0,051 * * * \\
(0,011)\end{array}$ & $\begin{array}{l}0,134 * * \\
(0,052)\end{array}$ & $\begin{array}{l}0,007 * * \\
(0,003)\end{array}$ & $\begin{array}{l}0,167 * * * \\
(0,047)\end{array}$ & $\begin{array}{l}0,009 * * * \\
(0,003)\end{array}$ \\
\hline $\begin{array}{l}\text { Betriebs- od. Per- } \\
\text { sonalrat }(1=\mathrm{ja})\end{array}$ & $\begin{array}{l}0,149 * * * \\
(0,052)\end{array}$ & $\begin{array}{l}0,040 * * * \\
(0,014)\end{array}$ & $\begin{array}{c}0,089 * \\
(0,049)\end{array}$ & $\begin{array}{c}0,024^{*} \\
(0,013)\end{array}$ & $\begin{array}{l}0,146^{* *} \\
(0,059)\end{array}$ & $\begin{array}{l}0,008 * * \\
(0,003)\end{array}$ & $\begin{array}{c}0,072 \\
(0,053)\end{array}$ & $\begin{array}{c}0,004 \\
(0,003)\end{array}$ \\
\hline $\begin{array}{l}\text { Tarifbindung } \\
(1=\mathrm{ja})\end{array}$ & $\begin{array}{c}0,077 * \\
(0,044)\end{array}$ & $\begin{array}{c}0,021 * \\
(0,012)\end{array}$ & $\begin{array}{c}0,064 \\
(0,041)\end{array}$ & $\begin{array}{c}0,017 \\
(0,011)\end{array}$ & $\begin{array}{l}0,096^{*} \\
(0,051)\end{array}$ & $\begin{array}{c}0,005^{*} \\
(0,003)\end{array}$ & $\begin{array}{c}0,061 \\
(0,045)\end{array}$ & $\begin{array}{c}0,003 \\
(0,002)\end{array}$ \\
\hline $\begin{array}{l}\text { Schriftlich fixierte } \\
\text { Pläne für die Per- } \\
\text { sonalentwicklung } \\
(1=\text { ja })\end{array}$ & $\begin{array}{l}0,471 * * * \\
(0,045)\end{array}$ & $\begin{array}{l}0,127 * * * \\
(0,012)\end{array}$ & $\begin{array}{l}0,454 * * * \\
(0,041)\end{array}$ & $\begin{array}{l}0,124 * * * \\
(0,011)\end{array}$ & $\begin{array}{l}0,547 * * * \\
(0,052)\end{array}$ & $\begin{array}{l}0,029 * * * \\
(0,003)\end{array}$ & $\begin{array}{l}0,527 * * * \\
(0,047)\end{array}$ & $\begin{array}{l}0,029 * * * \\
(0,003)\end{array}$ \\
\hline $\begin{array}{l}\text { Ausbildungsbe- } \\
\text { trieb }(1=\mathrm{ja})\end{array}$ & $\begin{array}{l}0,160 * * * \\
(0,049)\end{array}$ & $\begin{array}{l}0,043 * * * \\
(0,013)\end{array}$ & $\begin{array}{l}0,199 * * * \\
(0,046)\end{array}$ & $\begin{array}{l}0,054 * * * \\
(0,012)\end{array}$ & $\begin{array}{l}0,164 * * * \\
(0,056)\end{array}$ & $\begin{array}{l}0,009 * * * \\
(0,003)\end{array}$ & $\begin{array}{l}0,188 * * * \\
(0,050)\end{array}$ & $\begin{array}{l}0,010 * * * \\
(0,003)\end{array}$ \\
\hline $\begin{array}{l}\text { Logarithmierte } \\
\text { Beschäftigtenzahl }\end{array}$ & $\begin{array}{l}0,138 * * * \\
(0,020)\end{array}$ & $\begin{array}{l}0,037 * * * \\
(0,005)\end{array}$ & $\begin{array}{l}0,164 * * * \\
(0,018)\end{array}$ & $\begin{array}{l}0,045^{* * *} \\
(0,005)\end{array}$ & $\begin{array}{l}0,071 * * * \\
(0,022)\end{array}$ & $\begin{array}{l}0,004 * * * \\
(0,001)\end{array}$ & $\begin{array}{l}0,101 * * * \\
(0,019)\end{array}$ & $\begin{array}{l}0,006^{* * * *} \\
(0,001)\end{array}$ \\
\hline Branchendummies & $\mathrm{Ja}$ & & $\mathrm{Ja}$ & & $\mathrm{Ja}$ & & $\mathrm{Ja}$ & \\
\hline Pseudo $R^{2}$ & 0,168 & & 0,165 & & 0,100 & & 0,097 & \\
\hline Fallzahl & 6500 & & 7631 & & 6500 & & 7631 & \\
\hline Unzensierte Fälle & - & & - & & 994 & & 1200 & \\
\hline
\end{tabular}

Robuste Standardfehler in Klammern; Tobit-Modelle links- und rechtszensiert

Lesebeispiel (Modell 1, marginale Effekte): Erwartet ein Betrieb für die Zukunft Schwierigkeiten bei der Suche von Fachkräften, so erhöht dies die Wahrscheinlichkeit, dass er seine einfachen Beschäftigten weiterbildet, um 6,5 Prozentpunkte

Signifikanzniveau: $* * * p<0,01 ; * * p<0,05 ; * p<0,1$ 
Weiterbildungschancen einhergeht. Ein hoher Qualifiziertenanteil im Betrieb hat demgegenüber nur auf die Weiterbildungsintensität der einfach Beschäftigten einen signifikant positiven Effekt, der von der Größe her jedoch eher gering ist.

Die Wahrscheinlichkeit, dass ein Betrieb Weiterbildung für einfache Beschäftigte anbietet, sowie die Weiterbildungsbeteiligung dieser Mitarbeitergruppe werden zudem überwiegend positiv von den getätigten Investitionen beeinflusst. Dies deutet daraufhin, dass technische Neuerungen einen gewissen Anpassungs- und Qualifizierungsbedarf auch für die Gruppe der einfach Beschäftigten nach sich ziehen. Ein entsprechender positiver, wenn auch nicht besonders großer Effekt ist dabei insbesondere für Investitionen in Informations- und Kommunikationstechniken festzustellen. Hinsichtlich des technischen Standes der Anlagen (technische Ausstattung) kann hingegen kein signifikanter Effekt gefunden werden.

Ist ein Betrieb in einen Tarifvertrag eingebunden, so hat dies einen schwachen positiven Einfluss auf die Wahrscheinlichkeit, dass er seine einfach Beschäftigten weiterbildet. Manche Tarifverträge enthalten Regelungen zur Weiterbildung insgesamt bzw. zur Weiterbildung einzelner Mitarbeitergruppen (darunter auch Geringqualifizierte), was diesen Effekt erklären kann. Ebenso haben auch Betriebsräte bestimmte Mitspracherechte im Bereich der Weiterbildung, was sich empirisch in einem positiven Effekt der Betriebsratsvariable auf das Weiterbildungsangebot für einfach Beschäftigte niederschlägt. Auf die Intensität der Weiterbildung haben die Tarifbindung eines Betriebs sowie die Existenz eines Betriebsrats dahingegen nur in Modell 3, nicht aber in Modell 4 einen signifikanten Effekt.

Ist die Personalarbeit in einem Betrieb zu einem hohen Maße institutionalisiert (hier ausgedrückt durch das Vorliegen schriftlich fixierter Pläne zur Personalentwicklung), wirkt sich dies ebenfalls positiv auf die Weiterbildungschancen der Beschäftigten für einfache Tätigkeiten aus. Dabei erhöht sich die Wahrscheinlichkeit, dass ein Betrieb Weiterbildung für seine einfach Beschäftigten anbietet, um immerhin zwölf Prozentpunkte, wenn derartige Pläne vorhanden sind. Positiv mit der Weiterbildung einfach Beschäftigter geht zudem die Tatsache einher, dass es sich bei einem Betrieb um einen Ausbildungsbetrieb handelt, auch wenn der Effekt nicht besonders groß ausfällt.

Zur Beschreibung der uns besonders interessierenden Fachkräfteproblematik haben wir zwei verschiedene Indikatoren verwendet, und zwar zum einen die Erwartung von Schwierigkeiten bei der Fachkräfterekrutierung, und zum anderen das Vorliegen unbesetzter Stellen für Fachkräfte. Für beide Variablen ergibt sich ein signifikant positiver Effekt, d. h. Betriebe bilden dann verstärkt ihre Mitarbeitenden für einfache Tätigkeiten weiter, wenn sie bereits Probleme bei der Besetzung ihrer Fachkräftestellen haben oder solche für die Zukunft erwarten. Auch wenn dieser Effekt nicht besonders groß ausfällt (so erhöht das Vorliegen von unbesetzten Stellen die Weiterbildungswahrscheinlichkeit um fünf Prozentpunkte und die Erwartung von Fachkräfteproblemen um sieben Prozentpunkte), bestätigt der Befund dennoch unsere Hypothese, wonach auch einfach Beschäftigte ein wichtiges Potenzial zur Begegnung von Fachkräfteengpässen darstellen. Dabei ist allerdings zu beachten, dass die Daten des IAB-Betriebspanels keine Differenzierung der Art der Weiterbildung zulassen. Neben einer Entwicklungsqualifizierung, die auf höherwertige Tätigkeiten vorbereitet, kann es sich auch um eine Anpassungsqualifizierung handeln, 
wonach die Beschäftigten in der Form weitergebildet werden, dass sie anschließend veränderte Arbeitsanforderungen im eigenen Aufgabengebiet erfüllen können.

\section{Fazit}

Die Weiterbildung stellt für die Betriebe neben der Ausbildung eine weitere wichtige Säule zur Sicherung des Fachkräftebedarfs dar. Sind die gesuchten Qualifikationen auf dem externen Arbeitsmarkt nicht verfügbar, haben Unternehmen im Rahmen von Qualifizierungsmaßnahmen die Möglichkeit, Fachkräfteengpässen entgegenzuwirken. Untersuchungen zeigen, dass Unternehmen, die mit Fachkräfteproblemen zu kämpfen haben, tatsächlich stärker in der Weiterbildung insgesamt (Czepek et al. 2015), aber z. B. auch in der Weiterbildung älterer Beschäftigter engagiert sind, als Betriebe ohne derartige Probleme (vgl. u. a. Bellmann und Leber 2011). Wie die hier vorgelegten Analysen deutlich gemacht haben, gilt dies auch für die Weiterbildung von Beschäftigten für einfache Tätigkeiten. Hiernach stellen sich vor allem antizipierte Fachkräfteengpässe als Treiber der betrieblichen Weiterbildung von einfach Beschäftigten dar. Dabei ist allerdings einschränkend zu berücksichtigen, dass aufgrund der Operationalisierung von Weiterbildung im IAB-Betriebspanel nicht in jedem Fall davon ausgegangen werden kann, dass die Weiterbildung dieser Personengruppe auf ihre Qualifizierung für Fachkräftepositionen abstellt. Jedoch kann diese Personengruppe $-u$. a. aufgrund ihrer Weiterbildungserfahrung - für qualifizierte Tätigkeiten im Unternehmen gewonnen werden.

Auch wenn die Weiterbildungsbeteiligung von einfach Beschäftigten in Betrieben mit Fachkräfteschwierigkeiten höher ist als in Betrieben, die keine entsprechenden Probleme beklagen, ist diese Mitarbeitergruppe bei betrieblichen Weiterbildungsmaßnahmen nach wie vor unterrepräsentiert. In der Literatur werden für die geringe Weiterbildungsbeteiligung einfach Beschäftigter verschiedene Ursachen genannt: Während aus der Perspektive der Unternehmen Aspekte wie die Komplexität der Arbeitstätigkeit, aber auch der voraussichtliche Nutzen der Weiterbildungsteilnahme eine Rolle spielen, werden aufseiten der Beschäftigten Lernhemmnisse, bisherige negative (Lern-)Erfahrungen oder auch fehlende bedarfsgerechte Qualifizierungsangebote als mögliche Hürden zur Inanspruchnahme von Weiterbildungsmaßnahmen gesehen (Weber und Kretschmer 2012). Letztendlich sollten diese Hemmnisse über eine gezielte Einbindung der einfach Beschäftigten in betriebliche Weiterbildungsmaßnahmen überwunden werden können.

Zwar ist zu berücksichtigen, dass Weiterbildung kein Selbstzweck ist und es „offensichtlich (...) Menschen (gibt), die ihre Lebensaufgaben erfolgreich ohne jede Inanspruchnahme von Weiterbildungsangeboten bewältigen“ (Wittpoth 2011, S. 785). Gleichwohl ist eine geringe Teilnahme einfach Beschäftigter an Weiterbildung als kritisch zu sehen. Aus Sicht der Betriebe werden hierdurch wertvolle Potenziale zur Sicherung des Fachkräftebedarfs nicht ausgeschöpft, aus Sicht der einfach Beschäftigten bieten sich nur unzureichende Möglichkeiten zur Verbesserung der beruflichen Entwicklungs-, Einkommens- und Beschäftigungschancen. Insofern ist nach Strategien zu suchen, die zu einer Verbesserung der Weiterbildungschancen beitragen. Ein Ansatzpunkt kann dabei die Begegnung von etwaigen Lernhemmungen 
der Beschäftigten durch entsprechende Beratungsangebote und geeignete Lernformen sein. Wichtig erscheint in diesem Kontext auch ein kontinuierliches Lernen, beispielsweise im Rahmen einer lernförderlichen Arbeitsumgebung, damit das Lernen nicht verlernt wird und sich möglicherweise bereits bestehende Hürden weiter verstärken.

Ebenso erscheint es sinnvoll, die Betriebe für die Notwendigkeit und den Nutzen von Weiterbildungsmaßnahmen gerade auch für bildungsferne Gruppen (weiter) zu sensibilisieren und sie über entsprechende Angebote zu informieren. Wie unsere Analyse gezeigt hat, wirkt sich eine systematische Personalarbeit im Betrieb förderlich auf die Weiterbildung einfach Beschäftigter aus. Da gerade kleine und mittlere Unternehmen über eine solche aber oftmals nicht verfügen, ist hier ein weiterer Ansatz zu sehen.

Schließlich können Betriebe und Individuen auch durch finanzielle Fördermaßnahmen, die insbesondere auch die Gruppe der Geringqualifizierten im Fokus haben sollten, unterstützt werden. Denn letztlich ist der Fachkräftemangel auch ein gesellschaftliches Problem, das nur durch Aktivitäten auf verschiedenen Ebenen gelöst bzw. reduziert werden kann.

Open Access Dieser Artikel wird unter der Creative Commons Namensnennung 4.0 International Lizenz (http://creativecommons.org/licenses/by/4.0/deed.de) veröffentlicht, welche die uneingeschränkte Nutzung, Verbreitung und Wiedergabe für beliebige Zwecke erlaubt, sofern Sie den/die ursprünglichen Autor(en) und die Quelle ordnungsgemäß nennen, einen Link zur Creative Commons Lizenz beifügen und angeben, ob Änderungen vorgenommen wurden.

\section{Literatur}

Abel, J., Hirsch-Kreinsen, H., \& Ittermann, P. (2009). Einfacharbeit in der Industrie: Status Quo und Entwicklungsperspektiven. Dortmund: Technische Universität Dortmund.

Abel, J., Hirsch-Kreinsen, H., \& Ittermann, P. (2014). Einfacharbeit in der Industrie: Strukturen, Verbreitung und Perspektiven. Berlin: edition sigma.

Angrist, J. D., \& Pischke, J.-S. (2009). Mostly Harmless Econometrics. Princeton: Princeton University Press.

Becker, G. S. (1964). Human capital: A theoretical and empirical analysis with reference to education. Chicago: The University of Chicago Press.

Bellmann, L., \& Leber, U. (2011). Betriebliche Weiterbildung Älterer als Strategie zur Sicherung des Fachkräftebedarfs. Sozialer Fortschritt, 60(8), 168-175.

Bellmann, L., Grunau, P., Leber, U., \& Noack, M. (2013). Weiterbildung atypisch Beschäftigter. Gütersloh: Bertelsmann Stiftung.

Bundesministerium für Bildung und Forschung. (2013). Berufsbildungsbericht 2013. Bonn: BMBF.

Bundesministerium für Wirtschaft und Technologie. (2013). Engpassanalyse 2013. Besondere Betroffenheit in den Berufsfeldern Energie und Elektro sowie Maschinen- und Fahrzeugtechnik. Bonn: BMWi.

Clement, U. (2007). Kompetenz für einfache Arbeit? Anforderungen an Arbeit in modernen Produktionssystemen. In Friedrich-Ebert-Stiftung (Hrsg.), Perspektiven der Erwerbsarbeit: Einfache Arbeit in Deutschland (S. 35-45). Bonn: Friedrich-Ebert-Stiftung.

Czepek, J., Dummert, S., Kubis, A., Leber, U., Müller, A., \& Stegmaier, J. (2015). Betriebe im Wettbewerb um Arbeitskräfte. Bedarf, Engpässe und Rekrutierungsprozesse in Deutschland (IAB-Bibliothek 352). Bielefeld: W. Bertelsmann.

Düll, H. \& Bellmann, L. (1999). Der unterschiedliche Zugang zur betrieblichen Weiterbildung nach Qualifikation und Berufsstatus. Eine Analyse auf der Basis des IAB-Betriebspanels 1997 für West- und Ostdeutschland. Mitteilungen der Arbeitsmarkt- und Berufsforschung, 1, 70-84. 
Dummert, S., Kubis, A., Leber, U., \& Müller, A. (2014). Betrieblicher Arbeitskräftebedarf 2006-2012. IAB-Forschungsbericht, 23.

Ellguth, P., Kohaut, S., \& Möller, I. (2014). The IAB Establishment Panel - methodological essentials and data quality. Journal for Labour Market Research, 47(1/2), 27-41.

Elsholz, U. (2007). Nachqualifizierung von Erwerbspersonen ohne abgeschlossene Berufsausbildung (Vortrag auf dem 5. BIBB-Fachkongress im Forum 1, Arbeitskreis 2: Fachkräftebedarf und -entwicklung am 13. September 2007 in Düsseldorf).

Galiläer, L. (2006). Segmentierung von Anforderungsniveaus - die Erkenntnispotenziale von Qualifikationsentwicklungsforschung. Berufs- und Wirtschaftspädagogik online (bwpat), 11, 1-16. http://www. bwpat.de/ausgabe11/galilaeer_bwpat11.pdf. Zugegriffen: 26. März 2015.

Grunau, P. (2012). Betriebliche Berufsausbildung und Weiterbildung in Deutschland. http://datenreport. bibb.de/html/dr2012.html. Zugegriffen: 26. März 2015.

Hartung, S. (2013). Ausbildungsberechtigung, Ausbildungsaktivität und Übernahmeverhalten von Betrieben. In Bundesinstitut für Berufsbildung (Hrsg.), Datenreport zum Berufsbildungsbericht 2013. Informationen und Analysen zur beruflichen Bildung (S. 220-225). Bonn: BIBB.

Käpplinger, B. (2011). Motive und Motivationen in Unternehmen. In M. Kopel, C. Dunst, \& S. Saeed (Hrsg.), Weiterbildung in Berliner Betrieben (S. 54-70). Berlin: tredition.

Koalitionsvertrag zwischen CDU, CSU und SPD. (2013). Deutschlands Zukunft gestalten. 18. Legislaturperiode, 14. Dezember 2013. Berlin.

Köhler, C., \& Loudovici, K. (Hrsg.). (2007). Beschäftigungssysteme, Unsicherheit und Erwerbsorientierungen. Theoretische und empirische Befunde. SFB 580 Mitteilungen, 22.

Leber, U. (2009). Betriebsgröße, Qualifikationsstruktur und Weiterbildungsbeteiligung. Ergebnisse aus dem IAB-Betriebspanel. In F. Behringer, B. Käpplinger, \& G. Pätzold (Hrsg.), Betriebliche Weiterbildung. Der Continuing Vocational Training Survey (CVTS) im Spiegel nationaler und europäischer Perspektiven (Zeitschrift für Berufs- und Wirtschaftspädagogik: Beihefte, Bd. 22, S. 149-168).

Maier, T., Zika, G., Wolter, M. I., Kalinowski, M., \& Helmrich, R. (2014). Engpässe im mittleren Qualifikationsbereich trotz erhöhter Zuwanderung. BIBB-Report, 23.

Seyda, S., \& Werner, D. (2012). IW-Weiterbildungserhebung 2011 - Gestiegenes Weiterbildungsvolumen bei konstanten Kosten. IW-Trends, 1 .

Stegmaier, J. (2009). Betriebliche Berufsausbildung und Weiterbildung in Deutschland. http://datenreport. bibb.de/html/dr2010.html. Zugegriffen: 26. März 2015.

Troltsch, K. (2013). Berufliche Nachqualifizierung von zwei Millionen jungen Erwachsenen ohne Berufsabschluss - Welche Bereitschaft gibt es in Betrieben (BIBB-Fachbeitrag im Internet vom 27.05.2013). http://www.bibb.de/dokumente/pdf/a2_fachbeitrag-nachqualifizierung.pdf. Zugegriffen: 26. März 2015.

Weber, B., \& Kretschmer, S. (2012). Perspektiven beruflicher Weiterbildung für Geringqualifizierte. Wirtschaft und Beruf, 64(9/10), 39-44.

Williamson, O. (1990). Die ökonomischen Institutionen des Kapitalismus. Tübingen: Mohr.

Wittpoth, J. (2011). Beteiligungsregulation in der Weiterbildung. In R. Tippelt \& A. von Hippel (Hrsg.), Handbuch Erwachsenenbildung/Weiterbildung (4. Aufl., S. 771-788). Wiesbaden: VS Verlag für Sozialwissenschaften. 